Article

\title{
Association between Bone Biomarkers Osteoactivin and OPG with Plasma Levels of Irisin and Meteorin-like Protein in Peo- ple with T2D and Obesity
}

\author{
Preethi Cherian ${ }^{1 \sharp}$, Irina Al-Khairi1 ${ }^{\sharp}$, Mohammad Jamal ${ }^{3}$, Suleiman Al-Sabah ${ }^{4}$, Hamad Ali², Carol Dsouza ${ }^{3}$, Eman Al \\ Shawaf ${ }^{1}$, Waleed Al-Ali ${ }^{3}$, Ghanim Al-khaledi ${ }^{4}$, Fahd Al-Mulla ${ }^{2}$, Mohamed Abu-Farha ${ }^{1 *}$, Jehad Abubaker ${ }^{{ }^{*}}$
}

1 Department of Biochemistry and Molecular Biology, Dasman Diabetes Institute, Kuwait City 15462, Kuwait; irina.alkhairi@dasmaninstitute.org (I.A.); preethi.cherian@dasmaninstitute.org (P.C.); eman.alshawaf@dasmaninstitute.org (E.A)

2 Department of Genetic and Bioinformatics, Dasman Diabetes Institute, Kuwait City 15462, Kuwait; hamad.ali@HSC.EDU.KW (H.A.); fahd.almulla@dasmaninstitute.org (F.A.)

3 Department of Surgery, Faculty of Medicine, Health Sciences Centre, Kuwait University, Sulaibekhat 90805, Kuwait; mjamal110@gmail.com (M.J.); waleed mls@hotmail.com (W.A); ghanim.alkhaledi@ku.edu.kw (G. A.); cdcaroldsouza@gmail.com (C.D.)

${ }^{4}$ Department of Pharmacology \& Toxicology, Faculty of Medicine, Kuwait University, PO Box 24923, 13110 Safat, Kuwait; sulaiman.alsabah@ku.edu.kw (S.A)

* Correspondence: mohamed.abufarha@dasmaninstitute.org (M.A.-F.); jehad.abubakr@dasmaninstitute.org (J.A.); Tel.: +965-2224-2999 (M.A.-F. \& J.A.)

\begin{abstract}
The musculoskeletal system consisting of bones and muscles have been recognized as endocrine organs secreting hormones that are involved in regulating metabolic and inflammatory pathways. Obesity and type 2 diabetes (T2D) are associated with several musculoskeletal system complications. We hypothesized that an interaction exists between adipomyokines namely, irisin and METRNL, and various bone markers in individuals with obesity and T2D. A total of 228 individuals were enrolled in this study, including 124 non-diabetic and 104 T2D. A Multiplex assay was used to assess the level of various bone markers namely Osteoactivin, Syndecan, osteoprotegerin (OPG) and osteonectin/SPARC. Our data shows elevated levels of Osteoactivin, Syndecan, OPG and SPARC in T2D as compared to non-T2D individuals $(p \leq 0.05)$. Using Spearman's correlation, irisin was positively correlated only with Osteoactivin and OPG $(p<0.05)$. Similarly, a positive association was observed between METRNL and Osteoactivin $(p<0.05)$. The strong positive association shown in our study between irisin, METRNL and various bone markers emphasises the strong interaction between these organs. This suggests that a dysregulation in the functional interaction between these molecules could play a possible role in the development of bone and muscle related complications that are associated with obesity and T2D.
\end{abstract}

Keywords: Meteorin-like hormone, irisin, adipomyokines, bone markers, Osteoactivin, Syndecan, OPG, Osteonectin, type 2 diabetes, obesity.

\section{Introduction}

The musculoskeletal system consists mainly of bones and muscles that interacts physically and mechanically to support the body and allow its movement in addition to providing protection to vital organs. More recently, both bones and muscles have been recognized as endocrine organs secreting hormones that are involved in regulating metabolic and inflammatory pathways [1]. As a modern epidemic, diabesity is associated with metabolic dysregulation and chronic inflammation [2]. Obesity and diabetes are the cause of several complications associated with the musculoskeletal system including osteoporosis and sarcopenia [3,4]. Sarcopenia is defined as the progressive loss of muscle mass that is also associated with a decline in muscle function. Hallmarks of diabetes and metabolic diseases including insulin resistance, oxidative stress as well as lipid infiltration in 
muscles amongst the pathophysiological causes of sarcopenia [4]. On the other hand, Osteoporosis is a bone disorder that occurs when there is a decrease in bone density, increasing the risk of fracture. Diabetes has been strongly associated with an increased risk of osteoporosis-associated fractures [5]. Muscle and bone markers have been shown to be dysregulated in diabetes [6]. Our group has also previously reported an increase in the levels of two adipomyokines, irisin and Meteorin-like (METRNL) in T2D which was further exacerbated with obesity $[7,8]$.

Adipomyokines have recently been identified as molecules that are secreted by the skeletal muscle and adipose tissue and play an important role in modulating metabolic pathways [9-11]. Adipomyokines are released in response to several stimuli, including muscle contraction during physical activity and/or nutritional changes $[12,13]$. They have also been shown to mediate muscle growth (myogenesis) and regeneration. These molecules are part of a complex network that mediates crosstalk among muscles, liver, adipose tissue, brain and bone and they have also been shown to be involved in promoting beige fat thermogenesis [14].

Irisin and METRNL are two adipomyokines that have recently gained interest because of their role in increasing thermogenesis following exercise and cold exposure [15, 16]. Studies have also shown that irisin plays an important role in modulating bone metabolism through exercise [15]. Furthermore, Colaianni et. al. reported that irisin targets bone cells directly and enhances the differentiation of bone marrow stromal cells into mature osteoblasts [17]. In a follow up study by the same group it was demonstrated that injecting recombinant irisin (r-irisin) into mice lead to increased cortical bone mass and strength. This was attributed to a direct effect of irisin on osteoblastic bone formation, occurring mainly through suppressing a Wnt signalling inhibitor such as sclerostin (Sost) [18]. The beneficial effects of irisin were further shown in a study by Zhang et.al where the intraperitoneal (IP) administration of recombinant irisin induced osteoblastogenesis and inhibited osteoclastogenesis in bone cell lines. They reported that a threefold increase of irisin levels in circulation for 2 weeks reiterates the anabolic effects of exercise in the murine skeletal system [19]. Moreover, circulating serum level of irisin has been shown to be positively correlated with bone mineral status [20]. The adipomyokine METRNL was shown to have a unique expression pattern in bone and it was suggested that the anomalous expression of METRNL may inhibit bone cell differentiation [21].

Considering the involvement of adipomyokines in bone metabolism and the association between diabesity and bone loss, we aimed to investigate the potential link between irisin, METRNL and various markers of bone metabolism in people with obesity and T2D.

\section{Materials and Methods}

\subsection{Study population}

This study included 228 adult men and women with and without T2D that were further classified according to their body weight: 104 people with T2D (38 non-obese and 66 obese) and 124 people without T2D (73 non-obese and 51 obese). Diabetes was defined by fasting blood glucose $(\mathrm{FBG}) \geq 7 \mathrm{mmol} / \mathrm{L}$, receiving anti-diabetes treatment, or self-reported T2D [7, 22]. Obesity was defined according to body mass index (BMI); with BMI > $30 \mathrm{~kg} / \mathrm{m} 2$ considered obese and BMI between 20 and $30 \mathrm{~kg} / \mathrm{m} 2$ considered non-obese. A written informed consent was signed by all people willing to participate in the study. The study excluded people with morbid obesity (BMI $>40 \mathrm{~kg} / \mathrm{m} 2$ ) who had chronic conditions and/or were placed on medication/supplements known to influence body composition or bone mass. The study was approved by the ethical review board of Dasman Diabetes Institute and has been conducted in accordance with the ethical guidelines of the Declaration of Helsinki (Project\#: RA-2016-045).

\subsection{Blood sampling}


Venous blood samples were collected using Vacutainer EDTA tubes after fasting for a minimum of $8 \mathrm{~h}$. Collected blood was centrifuged at $400 \times \mathrm{g}$ for $10 \mathrm{~min}$ at room temperature and plasma was separated, aliquoted, and stored at $-80^{\circ} \mathrm{C}$ until the assay was performed.

\subsection{Measuring levels of METRNL and irisin in plasma.}

Plasma levels of METRNL and irisin were measured using the Enzyme Linked Immunosorbent Assay (ELISA). Plasma samples were thawed on ice and centrifuged for 5 min at $10,000 \times \mathrm{g}$ at $4^{\circ} \mathrm{C}$ to remove any remaining cells or platelets. Plasma level of METRNL was measured using the Human METRNL ELISA kit (LifeSpan BioSciences, Inc., Seattle, WA, USA. Cat\# LS-F13315). The samples were diluted 10× with sample diluent. ELISA was performed according to the manufacturer's instructions. The intra-assay coefficient of variation was $5.0 \%-10.0 \%$ while the inter-assay coefficient of variation was $<10 \%$. Plasma level of irisin was measured using the irisin recombinant ELISA kit (Phoenix Pharmaceuticals, Inc., Burlingame, CA, USA. Cat \#EK-067-29) following the manufacturer's kit instructions. Plasma samples were diluted $40 \times$ with the $1 \times$ assay buffer (provided in the kit). The intra-assay coefficient for this ELISA assay was $1.0 \%-7.0 \%$, while the inter-assay coefficient was $<20 \%$.

\subsection{Measuring the levels of bone markers in plasma}

Plasma levels of various bone markers namely: Osteoactivin, Syndecan, OPG and SPARC were assessed using a customized human premixed multi-analyte bone panel kit for Luminex assay (R\&D systems, Minneapolis, MN, USA. Cat\# LXSAHM). Samples were thawed on ice and centrifuged for $5 \mathrm{~min}$ at $10,000 \times \mathrm{g}$ at $4^{\circ} \mathrm{C}$ to remove any remaining cells or platelets. The level of the various bone markers was measured. Plasma was diluted $2 \times$ with the sample diluent and the assay was performed following the manufacturer's kit instructions. The assay was run on the Bio-Plex 200 system (Bio-Rad, CA, USA) and the Bio-Plex manager software was used to quantify the concentration of each analyte. The confidence level between the expected and observed concentration levels for each analyte was between $95-105 \%$ as assessed by the system.

\subsection{Statistical Analysis}

Student t-test was used to compare clinical data between T2D and non-diabetic people and between non-obese and obese people. The Mann-Whitney U test compared the plasma levels of METRNL and irisin. Spearman's correlation coefficient was used to determine the association between METRNL and irisin and to study their correlation with clinical and biochemical parameters. Data is reported as mean \pm standard error of mean (SEM). Statistical assessments were 2-sided and considered statistically significant at $p<$ 0.05. Analyses were performed using SAS software (version 9r; SAS Institute Inc., Cary, NC, USA).

\section{Results}

\subsection{Characteristics of the study population}

Selected characteristics of our sample population was studied by stratifying the population into 4 groups (non-diabetic: non-obese and obese, and T2D: non-obese and obese). The difference in BMI and waist/hip ratio was significantly higher in the non-diabetic obese people as compared to the non-diabetic non-obese people $(p<0.05)$. Moreover, the non-diabetic obese group demonstrated a significantly higher value of HOMA-B. However, the T2D obese group showed significantly higher FBG, and HbA1c when compared 
to the T2D non-obese group (Table 1). Circulating levels of irisin and METRNL were significantly higher in T2D and obesity, as shown in our previous report [7].

Table 1: Physical and biochemical characteristics of the non-diabetic and diabetic population categorized based on obesity

\section{Non-Diabetic}

$(n=124)$
Diabetic

$(n=104)$

\begin{tabular}{|c|c|c|c|c|c|c|}
\hline Phenotype & Non-Obese & Obese & $p$-value & Non-Obese & Obese & $p$-value \\
\hline & $(n=73)$ & $(n=51)$ & & $(n=38)$ & $(n=66)$ & \\
\hline Age (years) & $40.5 \pm 1.40$ & $43.64 \pm 1.77$ & 0.171 & $51.24 \pm 1.60$ & $52.91 \pm 1.11$ & 0.392 \\
\hline${ }^{+} B M I\left(k g / m^{2}\right)$ & $25.31 \pm 0.35$ & $34.04 \pm 0.45$ & $<0.001$ & $26.89 \pm 0.37$ & $34.34 \pm 0.30$ & $<0.001$ \\
\hline Waist/Hip ratio & $0.83 \pm 0.02$ & $0.89 \pm 0.01$ & 0.005 & $0.92 \pm 0.02$ & $0.98 \pm 0.03$ & 0.072 \\
\hline ‡TC $(\mathrm{mmol} / \mathrm{L})$ & $5.10 \pm 0.11$ & $5.09 \pm 0.14$ & 0.939 & $4.84 \pm 0.27$ & $4.93 \pm 0.14$ & 0.769 \\
\hline$\S$ HDL (mmol/L) & $1.39 \pm 0.05$ & $1.34 \pm 0.05$ & 0.478 & $1.23 \pm 0.10$ & $1.15 \pm 0.05$ & 0.435 \\
\hline "LDL (mmol/L) & $3.19 \pm 0.1$ & $3.21 \pm 0.13$ & 0.897 & $3.02 \pm 0.21$ & $3.0 \pm 0.13$ & 0.918 \\
\hline \$\$TGL (mmol/L) & $1.12 \pm 0.12$ & $1.214 \pm 0.10$ & 0.532 & $1.50 \pm 0.18$ & $1.71 \pm 0.15$ & 0.373 \\
\hline${ }^{++} \mathrm{FBG}(\mathrm{mmol} / \mathrm{L})$ & $5.23 \pm 0.15$ & $5.45 \pm 0.13$ & 0.267 & $7.21 \pm 0.38$ & $8.70 \pm 0.41$ & 0.009 \\
\hline 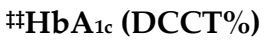 & $5.56 \pm 0.09$ & $5.57 \pm 0.08$ & 0.892 & $6.66 \pm 0.21$ & $8.23 \pm 0.22$ & $<0.001$ \\
\hline Insulin & $8.96 \pm 0.79$ & $9.58 \pm 0.91$ & 0.609 & $16.80 \pm 2.16$ & $14.13 \pm 1.37$ & 0.3 \\
\hline
\end{tabular}

Data are presented as mean \pm SEM. One-way ANOVA and post-hoc Bonferroni tests were used to compare various clinical and biochemical parameters (n=228). +BMI (body mass index); †TC (total cholesterol); §HDL (high-density lipoprotein); ILDL (low-density lipoprotein); §§TGL (triglycerides); ${ }^{+\dagger} \mathrm{FBG}$ (fasting blood glucose); ${ }^{\ddagger} \mathrm{Hb} \mathrm{H} 1 \mathrm{c}$ (hemoglobin A1c).

\subsection{Expression of Bone markers in circulation}

Measurement of circulating bone markers in plasma showed significantly higher levels of Osteoactivin in people with T2D $(20.614 \pm 0.588 \mathrm{ng} / \mathrm{mL})$ as compared to non-diabetic people $(16.591 \pm 0.298 \mathrm{ng} / \mathrm{mL}$ with $\mathrm{P}<0.0001)$ (Fig. 1A). A significant increase was also observed in OPG (T2D $=989.55 \pm 33.08$ and non-diabetic $=726.76 \pm 17.39 \mathrm{pg} / \mathrm{mL}$ with $\mathrm{P}<$ $0.0001)$ (Fig. 1B), SPARC (T2D $=1118.749 \pm 108.294$ and non-diabetic $=810.867 \pm 49.725$ $\mathrm{ng} / \mathrm{mL}$ with $\mathrm{P}=0.01)$ (Fig. $1 \mathrm{C})$ and Syndecan $(\mathrm{T} 2 \mathrm{D}=2075.57 \pm 97.93$ and non-diabetic= $1748.32 \pm 63.11 \mathrm{pg} / \mathrm{mL}$ with $\mathrm{P}=0.01$ ) (Fig. 1D). When the population was classified on the basis of obesity a significant increase was observed in the level of Osteoactivin (19.128 \pm $0.447 \mathrm{ng} / \mathrm{mL})$ and OPG $(890.43 \pm 28.28 \mathrm{pg} / \mathrm{ml})$ in obese as compared to non-obese people $(16.926 \pm 0.400 \mathrm{ng} / \mathrm{mL}$ and $752.87 \pm 20.90 \mathrm{pg} / \mathrm{ml}$ respectively) with $\mathrm{P}<0.0001$ (Fig. 2A \& 2B). Syndecan showed a trend of increase that did not reach significance. However, SPARC did not show any significant difference. Further classifying the population into the 4 groups, obese non-diabetic people had significantly higher plasma level of Osteoactivin $(17.363 \pm 0.499 \mathrm{ng} / \mathrm{mL})$ and OPG $(768.82 \pm 29.70 \mathrm{pg} / \mathrm{ml})$ when compared to non-obese non-diabetic people (Osteoactivin $=16.036 \pm 0.358 \mathrm{ng} / \mathrm{mL}$ and $\mathrm{OPG}=696.90 \pm 20.57 \mathrm{pg} / \mathrm{ml}$ ) 
with $\mathrm{P}=0.03$ and 0.04 respectively (Table 2 ). No significant difference was observed in the level of SPARC and Syndecan between non-diabetic obese and non-obese people (Table 2). Within the T2D population further stratification based on obesity did not show any significant difference in the level of all bone markers studied.

\section{Figure 1}

A
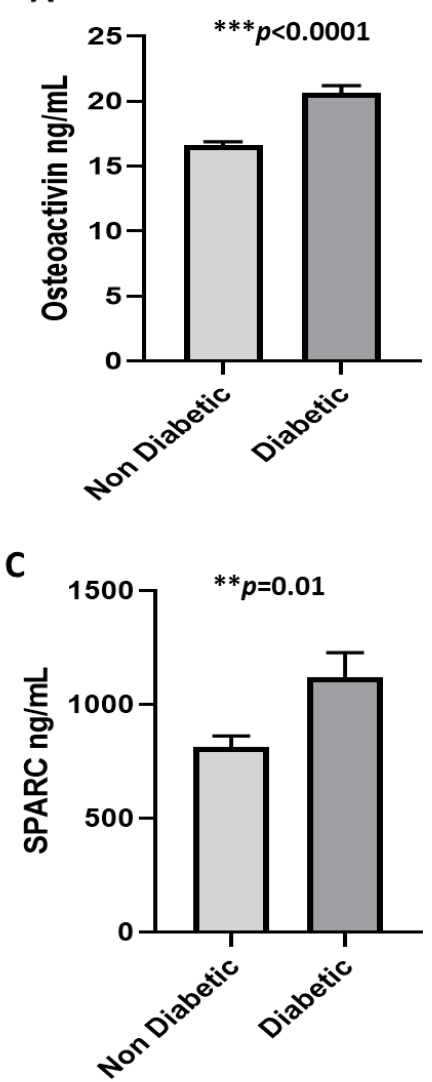

B

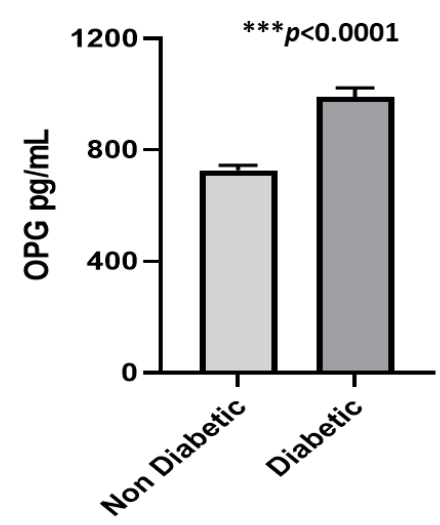

D

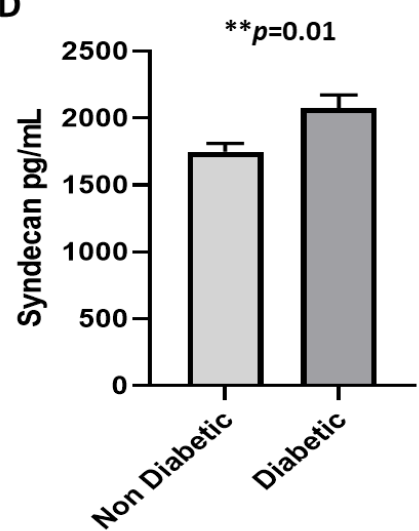

Figure 1. Bone marker level in plasma in all populations $(n=228)$. Comparing non-diabetic to T2D individuals. (A) Osteoactivin, (B) Osteoprotegerin (OPG), (C) Osteonectin (SPARK), (D) Syndecan. Levels of bone markers in plasma was determined using a multiplex bone panel. The population was classified based on their diabetic status. Diabetes was defined by fasting plasma glucose $\geq$ $126 \mathrm{mg} / \mathrm{L}(7 \mathrm{mmol} / \mathrm{L})$. Statistical assessment was 2-sided and considered statistically significant at ${ }^{*} p<0.05, * * p \leq 0.01, * * * p<0.001$. 
Figure 2

A

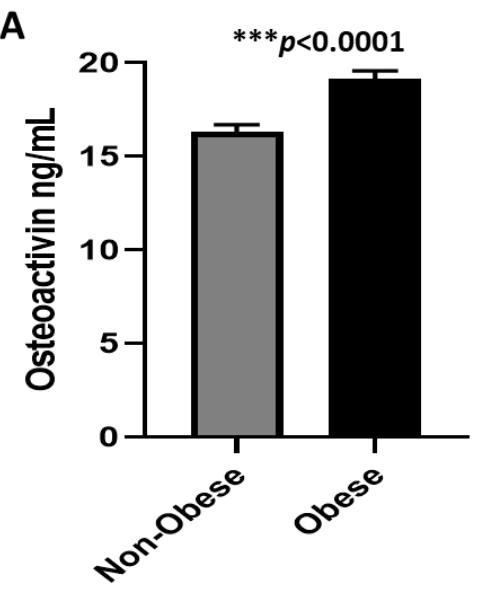

C

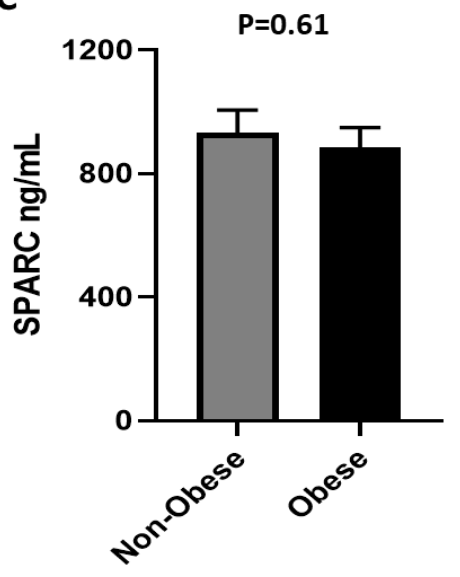

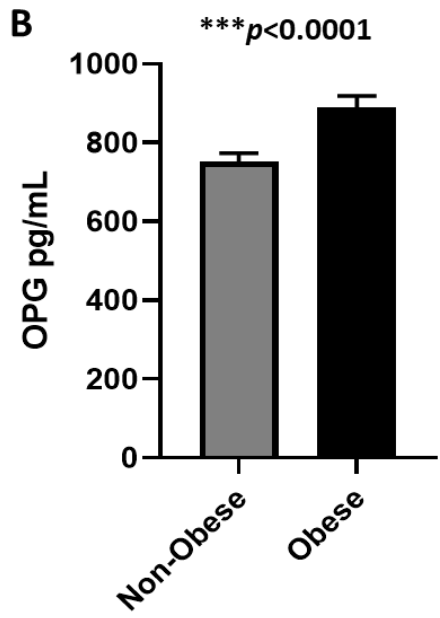

D

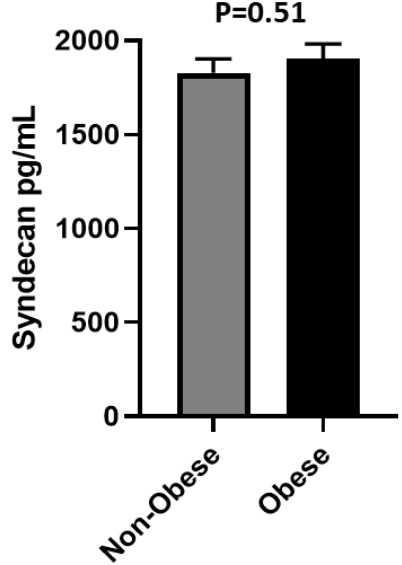

Figure 2. Bone marker level in plasma in all populations $(n=228)$. Comparing non-Obese to Obese individuals. (A) Osteoactivin, (B) Osteoprotegerin (OPG), (C) Osteonectin (SPARK), (D) Syndecan. Levels of bone markers in plasma was determined using a multiplex bone panel. The population was classified based on obesity. Obesity was defined based on BMI, where participants with BMI > $30 \mathrm{~kg} / \mathrm{m} 2$ were considered obese and those with BMI between 20 and $30 \mathrm{~kg} / \mathrm{m} 2$ were considered non-obese. Statistical assessment was 2 -sided and considered statistically significant at ${ }^{* * *} p<0.001$. 
Table 2: Plasma level of Bone markers in non-diabetic and diabetic population categorized based on obesity

\begin{tabular}{|c|c|c|c|c|c|c|}
\hline \multirow[b]{3}{*}{ Bone } & \multicolumn{3}{|c|}{ Non-Diabetic } & \multicolumn{3}{|c|}{ Diabetic } \\
\hline & \multicolumn{3}{|c|}{$(n=123)$} & \multicolumn{3}{|c|}{$(n=104)$} \\
\hline & Non-Obese & Obese & $p$-value & Non-Obese & Obese & $p$-value \\
\hline Markers & $(n=73)$ & $(n=50)$ & & $(n=38)$ & $(n=66)$ & \\
\hline Osteoactivin (ng/ml) & $16.036 \pm 0.358$ & $17.363 \pm 0.499$ & 0.03 & $19.54 \pm 1.067$ & $21.21 \pm 0.691$ & 0.19 \\
\hline OPG (pg/ml) & $696.90 \pm 20.57$ & $768.82 \pm 29.70$ & 0.04 & $912.80 \pm 45.88$ & $1034.33 \pm 44.27$ & 0.06 \\
\hline SPARC (ng/ml) & $795.04 \pm 71.33$ & $834.00 \pm 64.65$ & 0.68 & $1331.61 \pm 74.49$ & $957.92 \pm 133.71$ & 0.09 \\
\hline Syndecan (pg/ml) & $1680.65 \pm 79.69$ & $1842.31 \pm 02.01$ & 0.21 & $2256.03 \pm 43.08$ & $1972.03 \pm 129.43$ & 0.14 \\
\hline
\end{tabular}

Data are presented as mean \pm SEM. One-way ANOVA and post-hoc Bonferroni tests were used to compare various bone biomarkers $(\mathrm{n}=228)$.

\subsection{Correlation analysis}

We categorized the population based on their diabetes status; the correlation analysis was performed only on the non-diabetic population adjusted for age. This was done to exclude the effect of T2D related drugs. We observed a significant correlation between the levels of irisin and METRNL with Osteoactivin ( $\mathrm{r} 2=0.32$ and $\mathrm{P}<0.0001 ; \mathrm{r} 2=0.2$ and $\mathrm{P}=$ 0.01 respectively) (Fig $3 \mathrm{~A} \&$ Fig $4 \mathrm{~A}$ ). Irisin also showed a significant correlation with OPG (r2 $=0.21$ and $\mathrm{P}=0.01)$ (Fig 3B).

Figure 3
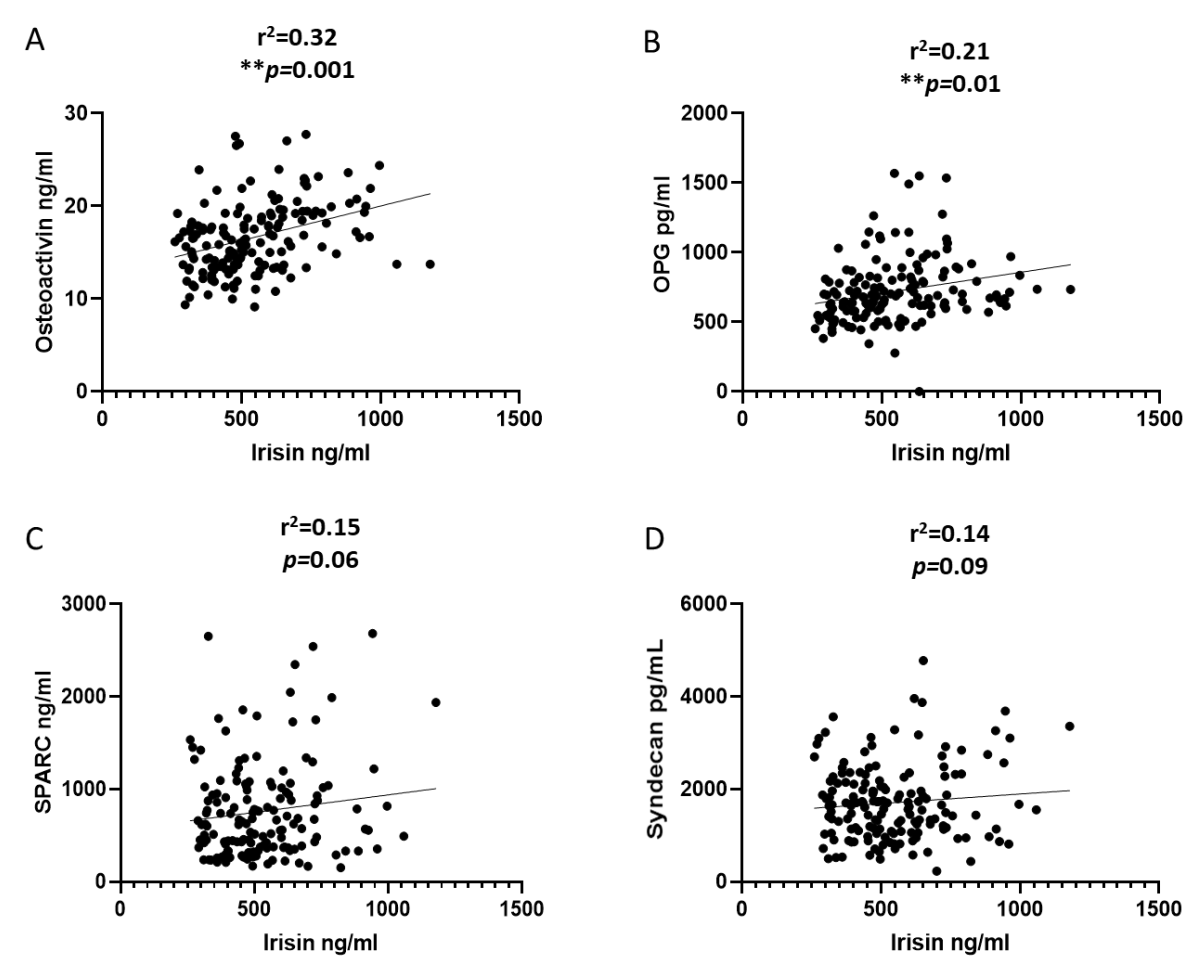
Figure 3. Correlation analysis between irisin and bone marker. (A) Osteoactivin, (B) Osteoprotegerin (OPG), (C) Osteonectin (SPARK), (D) Syndecan levels in plasma. Performed on the nondiabetic individuals age adjusted. Irisin level in plasma was determined using enzyme linked immunosorbent assay (ELISA), bone marker levels in plasma were determined using a multiplex bone panel. Diabetes was defined by fasting plasma glucose $\geq 126 \mathrm{mg} / \mathrm{L}$ ( $7 \mathrm{mmol} / \mathrm{L}$ ). Spearman correlation coefficient was used to determine the association of irisin with the specific bone markers. Statistical assessment was 2 -sided and considered statistically significant at $* * p \leq 0.01$.

\section{Figure 4}
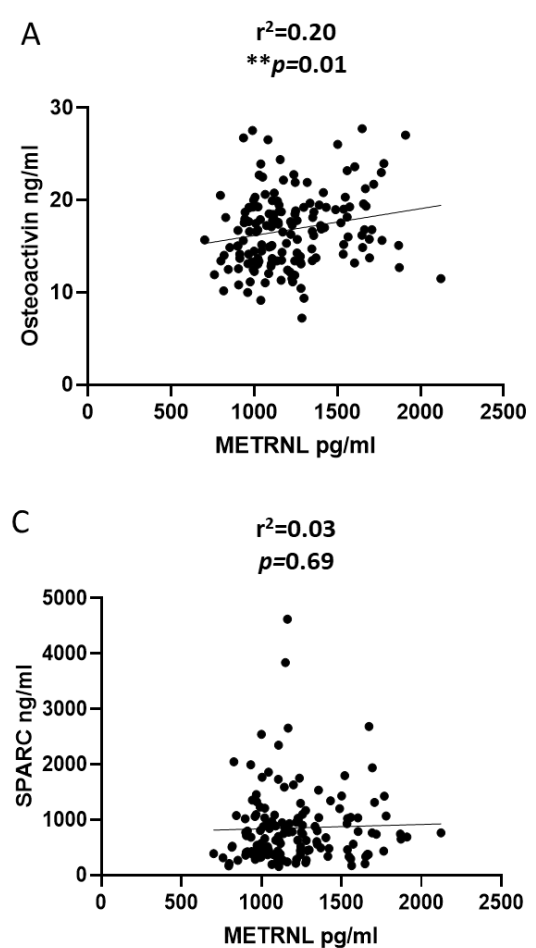
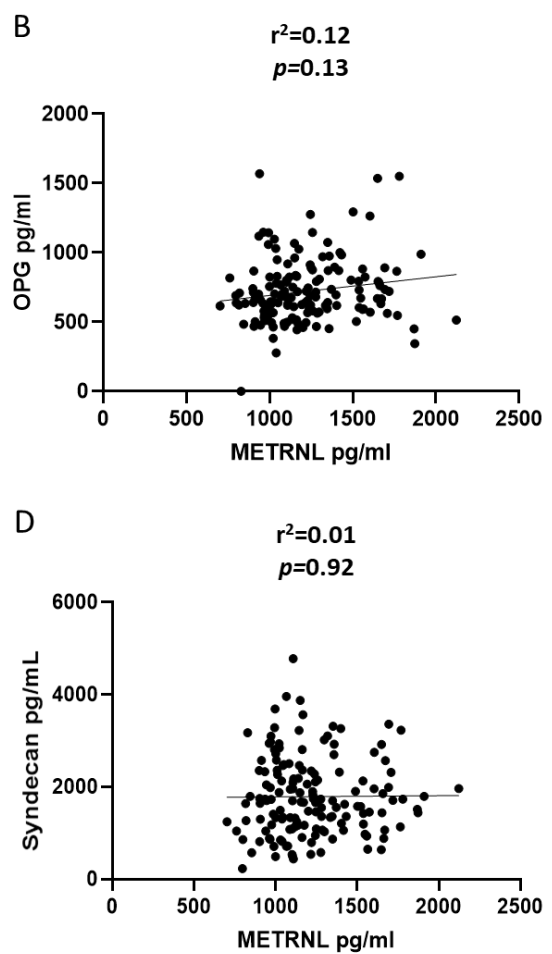

Figure 4. Correlation analysis between METRNL and bone markers. (A) Osteoactivin, (B) Osteoprotegerin (OPG), (C) Osteonectin (SPARK), (D) Syndecan levels in plasma. Performed on the non-diabetic individuals age adjusted. METRNL level in plasma was determined using enzyme linked immunosorbent assay (ELISA), bone marker levels in plasma were determined using a multiplex bone panel. Diabetes was defined by fasting plasma glucose $\geq 126 \mathrm{mg} / \mathrm{L}$ (7 mmol/L). Spearman correlation coefficient was used to determine the association of irisin with the specific bone markers. Statistical assessment was 2 -sided and considered statistically significant at ** $p \leq$ 0.01 .

\section{Discussion}

In this study we showed that plasma levels of Osteoactivin and OPG are increased with obesity and T2D concomitant with increased levels of irisin and METRNL. We also showed, for the first time, positive correlation between Osteoactivin and irisin and METRNL- the two main adipomyokines of our interest. Additionally, irisin showed a positive correlation with OPG. This association is particularly interesting as these bone markers are secreted by osteoblasts and play an important role in regulating the differentiation of bone cells [23].

Our previous studies have shown that the level of irisin and METRNL is increased with obesity and T2D implying that this may be a physiological attempt to restore glucose tolerance or a defense mechanism to counteract metabolic stress or resistance to these 
proteins, similar to insulin and leptin resistance [7]. Both Irisin and METRNL are primarily secreted by the skeletal muscles. It is known that there is a strong bone-muscle interaction and it has been reported that irisin plays an essential role in the interaction between muscle tissue and bone metabolism [10, 24, 25]. Recently there has been a stronger link between irisin and bone metabolism through the identification of an integ$\operatorname{rin} \alpha \mathrm{V} / \beta 5$ that acts as the irisin receptor for on osteocytes [26]. The authors of this study also showed that irisin treatment led to increased sclerostin level in osteocyte-like cells which contrasts with what was previously reported by Colaianni et al. where irisin level was negatively associated with sclerostin levels [27]. These conflicting data were thought to stem from a fundamental paradigm difference in the function of irisin like what is observed with the parathyroid hormone $(\mathrm{PTH})$ where it has both anabolic and catabolic effects on the skeleton [28]. It was postulated that increased level of irisin over an extended period can lead to increased bone catabolism while its knockout can lead to bone formation [28]. Additionally, our data shows a strong association between irisin, METRNL and two of the main bone remodelling factors namely, Osteoactivin and OPG. We suggest that this association may indicate a dysregulation in the functional interaction between these molecules that could play a possible role in the development of bone related complications associated with obesity and T2D. Our previous studies have shown that the level of irisin and METRNL is increased with obesity and T2D implying that this maybe a physiological attempt to restore glucose tolerance or a defense mechanism to counteract metabolic stress or resistance to these proteins, similar to insulin and leptin resistance[7].

Beyond its function in physically and mechanically supporting the body, the musculoskeletal system has been shown to secret hormones that are involved in regulating metabolic and inflammatory pathways. One such protein that plays an important role in bone metabolism is Osteoactivin, which is also known as GPNMB, is a membrane- bound protein that is highly glycosylated and localized on the cell surface or stored in endosomes/lysosomes. It can be cleaved to generate a soluble isoform [29]. This protein was originally discovered in melanoma cell lines [30], Osteoblasts [31] and dendritic cells [32]. Osteoactivin is also expressed in other cell types, such as osteoclasts, skin melanocytes and retinal pigment epithelial cells, hepatocytes, and leukocytes [33]. It is reported to be involved in a wide range of functions that are important in both physiological and pathological cellular processes. Osteoactivin is a positive regulator of osteoblast differentiation and has been recently identified as a regulator of lipogenesis in WAT. It was found to exacerbate diet induced obesity and insulin resistance [34]. It was also linked to obesity-driven inflammation in adipose tissue [35]. Microarray and transcriptomic analysis done on animal models revealed that Osteoactivin is one of the highly upregulated genes in fat tissues [36, 37]. Furthermore, Katayama et al, showed that overexpression of Osteoactivin in transgenic mice ameliorated fat accumulation and fibrosis in the liver in a diet-induced obesity model [38].

Osteoactivin affects adipose tissue macrophages, where it was shown to be highly expressed in macrophages within the adipose tissue of obese mice and humans [33]. Other reports have also shown that it is produced by macrophages and released in soluble form [39]. It is involved in suppressing T-cell activation as well as it suppresses the cytokine production [40]. Yu. et.al concluded that Osteoactivin positive macrophages negatively regulate inflammation as they observed that anti-inflammatory M2 macrophages express more Osteoactivin than the pro-inflammatory M1 macrophages [41]. Several other studies support the role of Osteoactivin in limiting inflammation and favoring tissue protection and repair at various sites (bone, kidney, and brain) [42, 43]. Therefore, we suggest that the overexpression of Osteoactivin observed in Obesity and T2D may be due to its possible role in combatting the adverse effects of inflammation associated with these conditions. Adipomyokines, such as irisin, are also known to play a role in maintaining the systemic low-grade inflammation during the progression of metabolic diseases. They are the key factors responsible for the skeletal muscle adaptive response to 
low-grade inflammation [24]. The association observed between osteoactivin and irisin highlights an important link between the two molecules especially that they cross interact in terms of their role in inflammation and insulin signaling.

On the other hand, OPG is a member of the tumor necrosis factor (TNF) receptor superfamily, originally characterized according to its ability to suppress osteoclast formation by preventing the binding of receptor activator of nuclear factor-kappa B ligand (RANKL) to receptor activator of NF-kappa B (RANK) on osteoclast precursor cells. OPG is initially produced as a glycosylated monomer of 55-62 kDa, which then undergoes homodimerization before being secreted as a disulfide-linked homodimer (the mature $110-120 \mathrm{kDa}$ form) $[44,45]$. The affinity of the OPG dimer for RANKL is much higher than that of the monomeric form [46]. OPG is expressed by many different cell types, including osteoclast precursors, mature osteoclasts, dendritic cells, B and T cells, fibroblasts, intestinal epithelial cells, vascular endothelial cells, and some cancer cells (e.g., breast and prostate cancers) [44]. The vascular role of OPG has gained much interest as it depends on the interplay with its ligands, RANKL and TRAIL, and a bidirectional modulation involving osteogenic, inflammatory and apoptotic responses. A study on OPG-deficient mice showed that these mice exhibited vascular calcification, suggesting a protective role of OPG, particularly in terms of vascular calcification [47]. Numerous clinical studies have consistently reported higher serum levels of OPG in association with cardiovascular outcome including coronary artery disease (CAD), vascular calcification, advanced atherosclerosis, diabetic complications, heart failure, abdominal aortic aneurysm, and cardiovascular mortality [48]. In a study done on Chinese Postmenopausal Women with Prediabetes and Type 2 Diabetes showed that serum OPG levels were significantly associated with HOMA-IR in the Chinese population and OPG levels were significantly higher in subjects with impaired glucose regulation. It is suggested that this increase in serum OPG levels is an insufficient compensatory self-defensive response to prevent vascular endothelial dysfunction and the progression of atherosclerosis [49]. Our study agrees with previous reports showing increased level of OPG in Obesity and T2D. It is however, still to be elucidated whether this overexpression of OPG protective or detrimental in the development and progression of vascular diseases which are closely linked to Obesity and T2D [50]. One of the main limitations of our current study is that it has a cross-sectional study design. This make it difficult to decipher the actual interaction between both irisin and METRNL with Osteoactivin and OPG. As a result, more functional analysis to better understand the interaction between these markers and establish their role in metabolic diseases need to be performed.

\section{Conclusions}

In conclusion, our findings suggest that there is a dysregulation in various markers in the musculoskeletal system mainly irisin, METRNL, Osteoactivin and OPG, which may result in the development of various complications associated with obesity and T2D. We also suggest that there is a possible cross-talk between adipomyokines and bone biomarkers that may play a possible role in the development of bone and muscle related complications that are associated with obesity and T2D. Further studies are necessary to understand the mechanistic significance of their possible interplay.

\section{Supplementary Materials: None}

Author Contributions: F.A.-M. and J.A. designed, helped in writing, reviewed the manuscript and
supervised the experiments. I.A., M.A.-F. and P.C. performed the experiments and wrote the man-
uscript. A.A., R.N. and M.M. participated in the data analysis. M.J., S.A.-S., H.A. and J.T. critically
reviewed the manuscript. I.A., M.A.-F. and P.C. contributed equally to this work. All the authors
reviewed the manuscript.

Funding: This research was funded by Kuwait Foundation for the Advancement of Sciences (KFAS), grant number RA-2016-045 
Institutional Review Board Statement: In this section, please add the Institutional Review Board Statement and approval number for studies involving humans or animals. Please note that the Editorial Office might ask you for further information. Please add "The study was conducted according to the guidelines of the Declaration of Helsinki, and approved by the Institutional Review Board (or Ethics Committee) of NAME OF INSTITUTE (protocol code XXX and date of approval)." OR "Ethical review and approval were waived for this study, due to REASON (please provide a detailed justification)." OR "Not applicable." for studies not involving humans or animals. You might also choose to exclude this statement if the study did not involve humans or animals.

Informed Consent Statement: Informed consent was obtained from all subjects involved in the study.

Data Availability Statement: In this section, please provide details regarding where data supporting reported results can be found, including links to publicly archived datasets analyzed or generated during the study. Please refer to suggested Data Availability Statements in section "MDPI Research Data Policies" at https://www.mdpi.com/ethics. You might choose to exclude this statement if the study did not report any data.

Acknowledgments: We would like to thank the National Dasman Diabetes Biobank Core Facility at DDI for their contribution in sample processing. We are also indebted to Kuwait Foundation for the Advancement of Sciences (KFAS) for financial support of this research project (RA-20102016003045). The funding agency was not involved in data collection, analysis, or interpretation; trial design; patient recruitment; or any aspect pertinent to the study.

Conflicts of Interest: The authors declare that there is no potential conflicts of interest associated with this manuscript.

\section{References}

1. Magaro, M.S., et al., Identification of Sclerostin as a Putative New Myokine Involved in the Muscle-to-Bone Crosstalk. Biomedicines, 2021. 9(1).

2. 2. Wells, J.C.K., The diabesity epidemic in the light of evolution: insights from the capacity-load model. Diabetologia, 2019. 62(10): p. 1740-1750.

3. 3. Barazzoni, R., et al., Sarcopenic obesity: Time to meet the challenge. Clin Nutr, 2018. 37(6 Pt A): p. $1787-1793$.

4. 4. Trierweiler, H., et al., Sarcopenia: a chronic complication of type 2 diabetes mellitus. Diabetol Metab Syndr, 2018. 10: p. 25.

5. 5. Romero-Diaz, C., et al., Diabetes and Bone Fragility. Diabetes Ther, 2021. 12(1): p. 71-86.

6. 6. Jackuliak, P. and J. Payer, Osteoporosis, fractures, and diabetes. Int J Endocrinol, 2014. 2014: p. 820615.

7. 7. AlKhairi, I., et al., Increased Expression of Meteorin-Like Hormone in Type 2 Diabetes and Obesity and Its Association with Irisin. Cells, 2019. 8(10).

8. 8. Jamal, M.H., et al., Effect of sleeve gastrectomy on the expression of meteorin-like (METRNL) and Irisin (FNDC5) in muscle and brown adipose tissue and its impact on uncoupling proteins in diet-induced obesity rats. Surg Obes Relat Dis, 2020. 16(12): p. 1910-1918.

9. 9. Raschke, S. and J. Eckel, Adipo-myokines: two sides of the same coin--mediators of inflammation and mediators of exercise. Mediators Inflamm, 2013. 2013: p. 320724.

10. 10.Li, F., et al., Myokines and adipokines: Involvement in the crosstalk between skeletal muscle and adipose tissue. Cytokine Growth Factor Rev, 2017. 33: p. 73-82.

11. 11.Graf, C. and N. Ferrari, Metabolic Health-The Role of Adipo-Myokines. Int J Mol Sci, 2019. 20(24).

12. 12.Leal, L.G., M.A. Lopes, and M.L. Batista, Jr., Physical Exercise-Induced Myokines and Muscle-Adipose Tissue Crosstalk: A Review of Current Knowledge and the Implications for Health and Metabolic Diseases. Front Physiol, 2018. 9: p. 1307.

13. 13.Senesi, P., L. Luzi, and I. Terruzzi, Adipokines, Myokines, and Cardiokines: The Role of Nutritional Interventions. Int J Mol Sci, 2020. 21(21).

14. 14.Severinsen, M.C.K. and B.K. Pedersen, Muscle-Organ Crosstalk: The Emerging Roles of Myokines. Endocr Rev, 2020. 41(4).

15. 15.Buccoliero, C., et al., The effect of Irisin on bone cells in vivo and in vitro. Biochem Soc Trans, 2021. 49(1): p. 477-484.

16. 16.Rao, R.R., et al., Meteorin-like is a hormone that regulates immune-adipose interactions to increase beige fat thermogenesis. Cell, 2014. 157(6): p. 1279-1291.

17. 17.Colaianni, G., et al., Irisin enhances osteoblast differentiation in vitro. Int J Endocrinol, 2014. 2014: p. 902186.

18. 18. Colaianni, G., et al., The myokine irisin increases cortical bone mass. Proc Natl Acad Sci U S A, 2015. 112(39): p. 1215762.

19. 19.Zhang, J., et al., Exercise-induced irisin in bone and systemic irisin administration reveal new regulatory mechanisms of bone metabolism. Bone Res, 2017. 5: p. 16056. 
20. 20.Zhong, X., et al., The production, detection, and origin of irisin and its effect on bone cells. Int J Biol Macromol, 2021. 178: p. 316-324.

21. 21.Gong, W., et al., Meteorin-Like Shows Unique Expression Pattern in Bone and Its Overexpression Inhibits Osteoblast Differentiation. PLoS One, 2016. 11(10): p. e0164446.

22. 22. American Diabetes, A., Diagnosis and classification of diabetes mellitus. Diabetes Care, 2008. 31 Suppl 1: p. S55-60.

23. 23.Lee, K., et al., Roles of Mitogen-Activated Protein Kinases in Osteoclast Biology. Int J Mol Sci, 2018. 19(10).

24. 24.Gomarasca, M., G. Banfi, and G. Lombardi, Myokines: The endocrine coupling of skeletal muscle and bone. Adv Clin Chem, 2020. 94: p. 155-218.

25. 25. Estell, E.G., et al., Irisin directly stimulates osteoclastogenesis and bone resorption in vitro and in vivo. Elife, 2020.9.

26. 26.Kim, H., et al., Irisin Mediates Effects on Bone and Fat via alphaV Integrin Receptors. Cell, 2018. 175(7): p. 1756-1768 e17.

27. 27.Colaianni, G., et al., Irisin prevents and restores bone loss and muscle atrophy in hind-limb suspended mice. Sci Rep, 2017. 7(1): p. 2811.

28. 28.Colaianni, G., et al., Irisin and Bone: From Preclinical Studies to the Evaluation of Its Circulating Levels in Different Populations of Human Subjects. Cells, 2019. 8(5).

29. 29. Rose, A.A., et al., ADAM10 releases a soluble form of the GPNMB/Osteoactivin extracellular domain with angiogenic properties. PLoS One, 2010. 5(8): p. e12093.

30. 30.Weterman, M.A., et al., nmb, a novel gene, is expressed in low-metastatic human melanoma cell lines and xenografts. Int J Cancer, 1995. 60(1): p. 73-81.

31. 31.Safadi, F.F., et al., Cloning and characterization of osteoactivin, a novel cDNA expressed in osteoblasts. J Cell Biochem, 2001. 84(1): p. 12-26.

32. 32.Shikano, S., et al., Molecular cloning of a dendritic cell-associated transmembrane protein, DC-HIL, that promotes RGDdependent adhesion of endothelial cells through recognition of heparan sulfate proteoglycans. J Biol Chem, 2001. 276(11): p. 8125-34.

33. 33.Tsou, P.S. and A.H. Sawalha, Glycoprotein nonmetastatic melanoma protein B: A key mediator and an emerging therapeutic target in autoimmune diseases. FASEB J, 2020. 34(7): p. 8810-8823.

34. 34.Gong, X.M., et al., Gpnmb secreted from liver promotes lipogenesis in white adipose tissue and aggravates obesity and insulin resistance. Nat Metab, 2019. 1(5): p. 570-583.

35. 35.Atlas., T.H.P. 2020.

36. 36.Choi, M.S., et al., High-fat diet decreases energy expenditure and expression of genes controlling lipid metabolism, mitochondrial function and skeletal system development in the adipose tissue, along with increased expression of extracellular matrix remodelling- and inflammation-related genes. Br J Nutr, 2015. 113(6): p. 867-77.

37. 37.Zambonelli, P., et al., Transcriptional profiling of subcutaneous adipose tissue in Italian Large White pigs divergent for backfat thickness. Anim Genet, 2016. 47(3): p. 306-23.

38. 38. Katayama, A., et al., Beneficial impact of Gpnmb and its significance as a biomarker in nonalcoholic steatohepatitis. Sci Rep, 2015. 5: p. 16920.

39. 39.Liguori, M., et al., The soluble glycoprotein NMB (GPNMB) produced by macrophages induces cancer stemness and metastasis via CD44 and IL-33. Cell Mol Immunol, 2021. 18(3): p. 711-722.

40. 40.Sasaki, F., et al., Expression of glycoprotein nonmetastatic melanoma protein B in macrophages infiltrating injured mucosa is associated with the severity of experimental colitis in mice. Mol Med Rep, 2015. 12(5): p. 7503-11.

41. 41.Yu, B., et al., Macrophage-Associated Osteoactivin/GPNMB Mediates Mesenchymal Stem Cell Survival, Proliferation, and Migration Via a CD44-Dependent Mechanism. J Cell Biochem, 2016. 117(7): p. 1511-21.

42. 42.Zhou, L., et al., Glycoprotein non-metastatic melanoma protein b (Gpnmb) is highly expressed in macrophages of acute injured kidney and promotes M2 macrophages polarization. Cell Immunol, 2017. 316: p. 53-60.

43. 43.Kumagai, K., et al., Glycoprotein Nonmetastatic Melanoma B (Gpnmb)-Positive Macrophages Contribute to the Balance between Fibrosis and Fibrolysis during the Repair of Acute Liver Injury in Mice. PLoS One, 2015. 10(11): p. e0143413.

44. 44.Perez de Ciriza, C., A. Lawrie, and N. Varo, Osteoprotegerin in Cardiometabolic Disorders. Int J Endocrinol, 2015. 2015: p. 564934 .

45. 45.Sakamoto MK, T.R., Kiyoura Y, Beyond bone remodeling-emerging functions of osteoprotegerin in host defense and microbial infection. Integrative Molecular Medicine, 2015. 2(6): p. 384-390.

46. 46.Schneeweis, L.A., D. Willard, and M.E. Milla, Functional dissection of osteoprotegerin and its interaction with receptor activator of NF-kappaB ligand. J Biol Chem, 2005. 280(50): p. 41155-64.

47. 47. Bucay, N., et al., osteoprotegerin-deficient mice develop early onset osteoporosis and arterial calcification. Genes Dev, 1998. 12(9): p. 1260-8.

48. 48.Kiechl, S., et al., The osteoprotegerin/RANK/RANKL system: a bone key to vascular disease. Expert Rev Cardiovasc Ther, 2006. 4(6): p. 801-11.

49. 49.Duan, P., et al., Serum Osteoprotegerin Is a Potential Biomarker of Insulin Resistance in Chinese Postmenopausal Women with Prediabetes and Type 2 Diabetes. Int J Endocrinol, 2017. 2017: p. 8724869.

50. 50.Van Campenhout, A. and J. Golledge, Osteoprotegerin, vascular calcification and atherosclerosis. Atherosclerosis, 2009. 204(2): p. 321-9. 